\title{
Cooperation with Externalities and Uncertainty
}

\author{
Helga Habis · Dávid Csercsik
}

The final publication is available at Springer via http://dx.doi.org/10.1007/s11067-014-92653

\footnotetext{
Abstract We introduce a new solution concept to problems with externalities, which is the first in the literature to take into account economic, regulatory and physical stability aspects of network problems in the very same model. A new class of cooperative games is defined where the worth of a coalition depends on the behavior of other players and on the state of nature as well. We allow for coalitions to form both before and after the resolution of uncertainty, hence agreements must be stable against both types of deviations. The appropriate extension of the classical core concept, the Sustainable Core, is defined for this new setup to test the stability of allocations in such a complex environment.

A prominent application, a game of consumers and generators on an electrical energy transmission network is examined in details, where the power in- and outlets of the nodes have to be determined in a way, that if any line

This work was supported by the Hungarian Academy of Sciences under its Momentum Programme (LP-004/2010), by the fund KAP-1.2-14/001, and by the Hungarian National Fund (OTKA NF-104706, PD-101106). The authors thak László Á. Kóczy for the helpful discussions.

Helga Habis

Lund University, Department of Economics, P.O. Box 7082, S-220 07 Lund, Sweden

Tel.: +46 (0)462220000

Fax: +46 (0)4622241 18

E-mail: helga.habis@nek.lu.se

Dávid Csercsik

Pázmány Péter Catholic University, Faculty of Information Technology and Bionics, Práter U. 50/A 1083 Budapest, Hungary

Tel.: +36-1 8864700

Fax: +36-1 8864724

Centre for Economic and Regional Studies of the Hungarian Academy of Sciences,

Game Theory Research Group, Budaörsi U. 45, 1112 Budapest, Hungary

Tel.: +36-13092652

Fax: +36-1 3193136

E-mail: csercsik@itk.ppke.hu
} 
instantaneously fails, none of the remaining lines may be overloaded. We show that fulfilling this safety requirement in a mutually acceptable way can be achieved by choosing an element in the Sustainable Core.

Keywords partition function form games · uncertainty $\cdot$ core $\cdot$ sustainability · networks $\cdot$ game theory $\cdot$ externalities

\section{Introduction}

Cooperative game theory analyzes how the gains of cooperation is to be divided among the members of a coalition. The most famous solution to this problem is the core (Gillies, 1959). In a classical, static cooperative game it is implicitly assumed that players can make binding agreements regarding the allocation of the value of a coalition if they cooperated. In most real life situations the core is not applicable, since its assumptions are too restrictive. At the same time cooperative game theory is often criticized for not being able to incorporate externalities appropriately. In this paper we introduce a new class of transferable utility games, where we allow for dynamics, uncertainty and externalities as well, and propose an extended notion of the core as a solution to this game which relaxes its restrictive assumptions.

Dynamic cooperation, cooperative games with more than one time periods, has already been studied by a number of papers. The introduction of dynamics only, already raises a crucial question; namely what are the consequences of a current deviation for the future, or more precisely, how to define a profitable deviation? Different answers to this question lead to different solution concepts. Habis and Herings (2011a) give a comparison of all these concepts in a two-period general equilibrium setting and conclude that the Weak Sequential Core is the most satisfactory concept so far. Habis and Herings (2011b) introduce transferable utility games with uncertainty, define the Weak Sequential Core in this setup and give its characterization.

In many cases the value of a coalition may well depend on the behavior of the rest of the players, thus we may have externality among coalitions. These situations can be modeled by partition function form games, introduced by Thrall and Lucas (1963). They take the pessimistic approach that residual players aim to minimize the payoff of the deviators. This idea is in the spirit of the $\alpha$-core (Aumann and Peleg, 1960), defined for NTU-games, where it is assumed that a coalition deviates only if it gets a higher payoff irrespective of the induced partition of the rest of the players. Its complete opposite is the optimistic approach of the $\omega$-core (Shenoy, 1980), where deviators expect to be helped by residuals. A more rational reaction is applied in the $\gamma$-core (Chander and Tulkens, 1997), where the deviating coalition must face individually best responses. Here it is true again, that different assumptions regarding residual behavior may lead to further different solution concepts. Here we will concentrate on the Recursive Core by Kóczy (2007), since it is less sensitive to optimism or pessimism than the $\alpha$ - and $\omega$-cores, furthermore allows a rational 
residual response as the $\gamma$-core, but this response is general, endogenous and consistent with the solution of the main game. ${ }^{1}$

In this paper we will combine the above two directions of research, and allow for the presence of uncertainty and externalities at the same time by introducing the partition function form game with uncertainty (hereafter PFUgame). A PFU-game consists of two time periods, 0 and 1 . In period 1 one out of a finite number of states of nature may materialize and conditional on the state, the players are involved in a particular partition function form game. An outcome therefore specifies a payoff-partition configuration conditional on each possible state of nature. A utility function is then used to assign a utility level to each profile of state-contingent payoffs.

We are interested in the appropriate definition of the core of a PFU-game. In this setting coalitions (forming some partition) are allowed to form in both time periods. Stability requires that a suggested allocation cannot be blocked by any coalition at any period, i.e. both before and after the resolution of uncertainty. If one would allow for binding agreements (Ray and Vohra (1997) or Predtetchinski et al (2002)) in our stochastic setting, then the game would become formally equivalent to a non-transferable utility game. We, on the other hand, are interested in the case where agents cannot make such fully binding agreements. Instead, agents will not stick to their agreements concerning the future if after the resolution of uncertainty, they are better off when deviating. Hence, we only allow for self-enforcing agreements in the spirit of Ray (1989) and Habis and Herings (2011b). These considerations lead to the concept of the Sustainable Core. Extending the characterization of the Weak Sequential Core (Habis and Herings, 2011b); We say that an allocation belongs to the Sustainable Core only if - conditional on the state of nature - it belongs to the Recursive Core of the PFF-game related to that state, and moreover there is no coalition (forming some partition) in period 0 that can propose statecontingent Recursive Core elements of the game restricted to that coalition which gives its members higher utility.

An application of the PFU-game is the electrical energy transmission network, where consumers and generators need to find a stable allocation of power in- and outlets, taking into account possible line failures. Csercsik and Kóczy (2011) show that both negative and positive externalities exist in a static electrical energy transmission network game of generators and consumers, and therefore suggest the Recursive Core as a solution concept. Here we extend their example by the possibility of line failures in the grid, which creates the uncertainty. We show that the Sustainable Core can be used to find a solution to this extended game.

The organization of the paper is as follows. First we introduce the notation and the definition of the core for the PFF-game in Section 2 followed by the definition of the PFU-game and our solution concept in Section 3. The appli-

1 Further desirable properties are discussed in (Kóczy, 2006). 
cation to the electrical energy transmission network is analyzed in Section 4 in detail, finally Section 5 concludes.

\section{Preliminaries}

Let $N=\{1,2, \ldots, n\}$ be the set of players, and its non-empty subsets are the coalitions, denoted by $C$. A cooperative game with transferable utility, or characteristic function form game, is a pair $(N, v)$, where $v: 2^{N} \rightarrow \mathbb{R}$ is a characteristic function which assigns to each coalition $C \subseteq N$ its worth $v(C)$, with the convention that $v(\emptyset)=0$.

\subsection{Partition Function Form Games}

A partition $P$ is a set of disjoint coalitions; $P=\left\{C^{1}, C^{2}, \ldots, C^{m}\right\}$, where their union is $N$; i.e., players in set $S$ cooperate if and only if $S \in P$. The set of partitions is $\mathcal{P}$ and the set of partitions of $C \subset N$ is $\mathcal{P}(C)$, with a typical element $P(C)$. A partition function; $w: \mathcal{P} \rightarrow\left(2^{N} \rightarrow \mathbb{R}\right)$ assigns a characteristic function to each partition. A cooperative game with transferable utility in partition function form, or briefly PFF-game, is a pair $(N, w)$, and it is an extension of the characterization function form game where we allow for externalities across players and coalitions; hence, the worth of a coalition may be different in each partition. For $C \in P$, let the worth $w(C, P)$ denote the amount that the players in $C$ can guarantee themselves by cooperating, when the coalition $C$ is embedded in the partition $P$. An outcome is a pair $(x, P)$, consisting of a payoff vector $x=\left(x^{1}, \ldots, x^{n}\right) \in \mathbb{R}^{N}$ satisfying feasibility; $\sum_{i \in C} x^{i} \leq w(C, P)$ for all $C \in P$, and a partition $P \in \mathcal{P}$. The payoff for a coalition $C$ is a vector $x^{C}=\left(x^{i}\right)_{i \in C} \in \mathbb{R}^{C}$. Let us denote the the set of outcomes in $(N, w)$ by $\Omega(N, w)$.

In a PFF-game whether a coalition has a profitable deviation, depends on the induced partition of the players. There are a number of ways to model the reaction of the remaining players, which lead to different solution concepts. Here we concentrate on the Recursive Core by Kóczy (2007), that allows the remaining, residual players to freely react, and form a core-stable partition before the payoff of the deviating coalition is evaluated. Thus, given a deviation, the residual players face the problem of solving another, smaller PFF-game. We call this a residual game.

Definition 1 Consider a game $(N, w)$ and a player set $R \subsetneq N$. Assume $\bar{R}=N \backslash R$ have formed the partition $P(\bar{R}) \in \mathcal{P}(\bar{R})$. Then the residual game $\left(R, w^{P(\bar{R})}\right)$ is the PFF game over the player set $R$ with the partition function given by $w^{P(\bar{R})}(C, P(R))=w(C, P(R) \cup P(\bar{R}))$.

Observe that the subset of players in the residual game still experience the externalities from the players in $\bar{R}$. Hence, if the core is the solution for $(N, w)$, then the core solves the residual game $\left(R, w^{P(\bar{R})}\right)$ as well. The definition of the 
residual game is similar to that of the reduced game (Moulin, 1985), where the payoff structure of the larger game is accounted for, but the significance of the partition of $\bar{R}$ is not considered.

Next we define the Recursive Core in the spirit of (Kóczy, 2007).

Definition 2 ((Pessimistic) Recursive Core) For a single-player game the (Pessimistic) Recursive Core is trivially defined. Now assume that the (Pessimistic) Recursive Core $\mathrm{RC}_{-}(N, w)$ has been defined for all games with $|N|<k$ players. For an $|N|$-player game an outcome $(x, P)$ is dominated if there exists a coalition $Q$ and a feasible payoff vector $y^{Q} \in \mathbb{R}^{Q}$, such that for all $\left(y^{Q}, y^{\bar{Q}}, Q \cup P(\bar{Q})\right) \in \Omega(N, w)$ we have $y_{i}>x_{i}$ for all $i \in Q$ and if $\mathrm{RC}_{-}\left(\bar{Q}, w^{Q}\right) \neq \varnothing$ then $\left(y^{\bar{Q}}, P(\bar{Q})\right) \in \mathrm{RC}_{-}\left(\bar{Q}, w^{P(Q)}\right)$. The (Pessimistic) Recursive Core $\mathrm{RC}_{-}(N, w)$ of $(N, w)$ is the set of undominated outcomes.

Definition 3 ((Optimistic) Recursive Core) For a single-player game the (Optimistic) Recursive Core is trivially defined. Now assume that the (Optimistic) Recursive Core $\mathrm{RC}_{+}(N, w)$ has been defined for all games with $|N|<k$ players. For an $|N|$-player game an outcome $(x, \mathcal{P})$ is dominated if there exists a coalition $Q$ and an outcome $(y, P(Q) \cup P(\bar{Q})) \in \Omega(N, w)$, such that $y_{i}>x_{i}$ for all $i \in Q$ and if $\mathrm{RC}_{+}\left(\bar{Q}, w^{P(Q)}\right) \neq \varnothing$ then $\left(y^{\bar{Q}}, P(\bar{Q}) \in \mathrm{RC}_{+}\left(\bar{Q}, w^{P(Q)}\right)\right.$. The Optimistic Recursive Core $\mathrm{RC}_{+}(N, V)$ of $(N, w)$ is the set of undominated outcomes.

In the following we will use the pessimistic approach for the Recursive Core. The Recursive Core is always well-defined. Note that any Recursive Core element consists of a stable payoff allocation and a corresponding stable partition of the players.

According to Definition 2 a deviating coalition $\bar{Q}$ has to evaluate the profit it can achieve based on the outcome of the residual game, where a rational residual behavior is expected. We assume conservatism of the deviators only among the remaining possible outcomes in the following sense. If the Recursive Core of the residual game is nonempty, then the members of coalition $\bar{Q}$ expect the lowest possible payoffs of the stable outcomes $(y, \bar{Q} \cup P(Q)) \in \Omega(N, w)$ with $\left(y^{Q}, P(Q)\right) \in \operatorname{RC}\left(Q, w^{P(\bar{Q})}\right)$. If the Recursive Core of the residual game is empty, then the payoff of the members of the deviating coalition must be higher than the given one regardless of the coalition structure of the residual players.

Based on the concept of the Recursive Core, a minimal claim function can be defined, which describes the minimal claim of each coalition in the corresponding PFF game reduced to that coalition. This function, termed $v^{m c}$ in the following, may be applied in the same spirit as a characteristic function, since it assigns a unique value to each coalition, which they can secure for themselves if they deviated. The formal definition of $v^{m c}$ is as follows.

Definition 4 Let us consider the residual game $\left(\bar{C}, w^{C}\right)$ over the player set $\bar{C}$ defined by the partition function $w^{C}\left(K, P^{\bar{C}}\right)=w\left(K, P^{\bar{C}} \cup C\right)$ where $K \in P^{\bar{C}} \in$ 
$\mathcal{P}(\bar{C})$. Let us denote the Recursive Core of the residual game by $\operatorname{RC}\left(\bar{C}, w_{C}\right)$. The minimal claim function $v^{m c}$ can be defined as

$$
v^{m c}(C)=\left\{\begin{array}{lll}
\min _{\sum_{i \in C} x^{i}}\left\{\Omega(N, w) \mid\left(x, P^{\bar{C}}\right) \in \mathrm{RC}\left(\bar{C}, w^{C}\right)\right\} & \text { if } & \mathrm{RC}\left(\bar{C}, w^{C}\right) \neq \emptyset \\
\min _{\sum_{i \in C} x^{i}}\{\Omega(N, w)\} & \text { if } & \operatorname{RC}\left(\bar{C}, w^{C}\right)=\emptyset
\end{array}\right.
$$

where $v^{m c}(C)$ is the minimal claim of coalition $C$.

With the help of the minimal claim function, a characterization of the Recursive Core can be given as follows.

Lemma 1 The Recursive Core $R C(N, w)$ of the game $(N, w)$ is a collection of outcomes $(x, P) \in \Omega(N, w)$, such that there is no coalition $C$ with $v^{m c}(C)>$ $\sum_{i \in C} x^{i}$

Proof Holds by construction.

\section{Games with Uncertainty}

We model uncertainty in a dynamic setup; we consider a game with two periods, $t \in T=\{0,1\}$. In period 1 , one state of nature $s$ out of a finite set of states of nature $S$ occurs. We define the state of nature for period 0 as state 0 , so the set of all states is $S^{\prime}=\{0\} \cup S$. Our interest is clearly in the cases with non-degenerate uncertainty; $|S|>1$. In period 1 the players are involved in a PFF-game $\Gamma_{s}=\left(N, w_{s}\right)$, where the game itself is allowed to be state-dependent. Period 0 serves as a point in time prior to the resolution of uncertainty.

Player $i \in N$ evaluates his payoffs by a utility function $u^{i}: \mathbb{R}^{S} \rightarrow \mathbb{R}$, which assigns to every profile of payoffs $x^{i}=\left(x_{1}^{i}, \ldots, x_{S}^{i}\right) \in \mathbb{R}^{S}$ a utility level $u^{i}\left(x^{i}\right)$ and is assumed to be continuous and state-separable, i.e. $u^{i}\left(x^{i}\right)=\sum_{s \in S} u_{s}^{i}\left(x_{s}^{i}\right)$, where $u_{s}^{i}\left(x_{s}^{i}\right)$ is monotonically increasing. A well-known example of utility functions satisfying these assumptions is the von NeumannMorgenstern utility function.

\subsection{The Model}

A PFF-game with uncertainty is defined as follows.

Definition 5 A partition function form game with uncertainty (PFU-game) $\Gamma$ is a tuple $\left(N, S^{\prime}, w, u\right)$ where $w=\left(w_{1}, \ldots, w_{S}\right)$ and $u=\left(u^{1}, \ldots, u^{n}\right)$.

Note that state 0 merely serves as a point in time where players face future uncertainty and may decide to cooperate (to pool risk for instance).

The outcome of the game is an ordered pair $(x, P)$ where the matrix $x=$ $\left(x^{1}, \ldots, x^{n}\right) \in \mathbb{R}^{S \times N}$ is called an allocation, and $P=\left(P_{1}, \ldots, P_{s}\right)$ a partition 
for each subgame. Let $\Omega(x, P)$ denote the set of outcomes in $\Gamma$. The state$s$ component $x_{s}=\left(x_{s}^{1}, \ldots, x_{s}^{n}\right) \in \mathbb{R}^{N}$ of an allocation is referred to as the allocation in state $s \in S$. The set of partitions in $\Gamma_{s}$ is $\mathcal{P}_{s}$ and the set of partitions in $\Gamma_{s}$ of $C \in N$ is $\mathcal{P}_{s}(C)$ with a typical element $P_{s}^{C}$. Let $\Gamma_{s}^{R}$ denote the residual PFF-game played in state $s$ by player set $R$. The payoff allocation matrix must satisfy a feasibility constraint; given the partition $P$, for all $C \in P$ we have $\sum_{i \in C} x_{s}^{i} \leq w_{s}(C, P)$ for all $s \in S$. The central question of a PFUgame is finding a stable outcome.

We study which outcomes of the game $\Gamma$ are stable. In general an outcome is stable if no coalition ever can benefit from a deviation. That is, we require that there should be no blocking possible in the subgames in $t=1$ or before the resolution of the uncertainty in $t=0$.

Thus we will require from a stable outcome $(x, P)$ of the game $\Gamma$ that each of its components $\left(x_{s}, P_{s}\right)$ belongs to the Recursive Core of the subgame $\Gamma_{s}$ to prevent any future deviation.

Now we are ready to define the Sustainable Core by means of the Recursive Core of suitably chosen subgames.

Definition 6 (Sustainable Core) An outcome $(x, P)$ belongs to the Sustainable Core of the game $\Gamma$, denoted by $\mathrm{SC}(\Gamma)$, if and only if

(a) $(x, P)$ is such that $\left(x_{s}, P_{s}\right) \in \mathrm{RC}\left(\Gamma_{s}\right)$ for all $s \in S$,

(b) and there is no coalition $R \in N$ forming some partition $\tilde{P}(R) \in \mathcal{P}(R)$ and outcome $(\tilde{x}(R), \tilde{P}(R))$ such that $\left(\tilde{x}(R)_{s}, \tilde{P}(R)_{s}\right) \in \operatorname{RC}\left(\Gamma_{s}(R)\right)$ for all $s \in S$, and $u^{i}\left(\tilde{x}^{i}\right)>u^{i}\left(x^{i}\right)$ for all $i \in R$.

It means that for an outcome to belong to the Sustainable Core of the PFU-game $\Gamma$, the outcome should belong to the Recursive Core of the PFFgame $\Gamma_{s}$ in every state $s \in S$. Moreover, no coalition (forming some partition) should be able to pick an element of the Recursive Core of the game restricted to $R$ in every state, and in doing so improve utility in an ex ante sense. Since we require the deviating coalition to pick a Recursive Core element, no further counter-deviation from it can be expected by any sub-coalition; i.e. the blocking allocation is indeed self-enforcing.

Note, that if there is no externality, the game boils down to a transferable utility game with uncertainty, and so the definition of the Sustainable Core will coincide with that of the Weak Sequential Core. Similarly, if there is no uncertainty, than we have a single PFF-game with the Recursive Core as the solution to it.

In a PFU-game one can distinguish ex ante and ex post efficiency.

Definition 7 An outcome $(\bar{x}, \bar{P})$ is ex ante efficient in the game $\Gamma$ if:

(i) $\sum_{i \in N} \bar{x}^{i} \leq w(N, \bar{P})$, and

(ii) there does not exist an outcome $(x, P)$ with $\sum_{i \in N} x^{i} \leq w(N, P)$ such that $u^{i}\left(x^{i}\right)>u^{i}\left(\bar{x}^{i}\right)$ for all $i \in N$.

Definition 8 An outcome $(\bar{x}, \bar{P})$ is ex post efficient in the game $\Gamma$ if $\sum_{i \in N} \bar{x}^{i}=$ $w(N, \bar{P})$. 
Note, that the concept of ex post efficiency says more than the usual feasibility conditions in TU-games, since it requires $\sum_{i \in N} \bar{x}_{s}^{i}=w_{s}(N, \bar{P})$ to hold at all states $s \in S$, but contrary to ex ante efficiency it does not imply Pareto efficiency, since it does not consider reallocation possibilities across states.

The next observation follows from the Pareto efficiency of the Recursive Core.

Corollary 1 If $(\bar{x}, \bar{P}) \in \mathrm{SC}(\Gamma)$ then $(\bar{x}, \bar{P})$ is ex post efficient.

Note, on the other hand, that outcomes need not be ex ante efficient. Since binding agreements are not allowed for here, certain utility transfers across states might not be feasible.

\section{Application: Safety Critical Rescheduling of Generators in The Electrical Power Transmission Network}

The modeling of electricity markets (see eg. Kleindorfer et al (2001); Smeers (2003); Metzler et al (2003); De la Torre et al (2003); Ehrenmann (2004); Gabriel et al (2004); Oggioni et al (2012); Abrell and Weigt (2012); Leuthold et al (2012)) has always been one of the most challenging and complex tasks due to the special physical, engineering and economical aspects of the field. The liberalization of the electricity markets, and the privatization and restructuring of power systems in the last decades resulted in an increased effort to fully economically exploit the infrastructure of the power grid. With this trend, the risk of failures has increased as demonstrated by the large blackouts observed (Fairley, 2004; Hines et al, 2009). As emphasized by Beccuti et al (2010), the situation is complicated by the fact that electrical power grids are large interconnected systems covering large areas where the failures of components or control actions of a regional operator may adversely affect other components located hundreds of kilometers away. This observation suggests that the problem can be modeled by a PFF-game.

To avoid such dramatic consequences as country-wide blackouts, power system operators use various methodologies to keep the power grid in safe operational state. The protocols applied in these cases of security arrangements may affect the electricity market.

In this application we analyze, how the possibly occurring emergency procedures may be taken into account by the various stakeholders of the electricity market during the planning of transmission contracts. For the aim of simplicity we consider only line failures (no generator failures). We have to note that the method demonstrated here, the application of a PFU-game, can be straightforwardly applied to other scenarios of uncertainty; for example to network expansion scenarios, in which the uncertain expansion (the possible addition of certain lines) is assumed to be (partially) independent of the market participants, who represent the players of the transmission game. 


\subsection{PFF-game on The Transmission Network}

Previously, a PFF-game for the electrical transmission networks has been defined in (Csercsik and Kóczy, 2011). The most important features of the applied transmission model are the following. The admittance values $(Y)$ and the injected/consumed energy amounts of the nodes uniquely determine the energy flows on the edges (branches or lines) of the network, which can be obtained by the solution of a system of linear equations. In addition to its admittance value, each edge is characterized by a transfer capacity $(\bar{q})$, which corresponds to the maximal amount of energy which can be transferred on it. Furthermore, as a most simple approach we neglect transmission losses. For the sake of simplicity we will assume that every node of the energy transmission network corresponds to a certain generator or consumer.

If we consider a network with $n$ nodes (generators or consumers) and $m$ edge, line flows (Q) of a DC load flow model are determined as

$$
Q=B^{D} A^{T} B^{+} P
$$

where $P$ is the vector of the generated/consumed power at the nodes, $A \in$ $\mathbb{R}^{n \times m}$ is the the node-branch incidence matrix of the network, $B \in \mathbb{R}^{n \times n}$ denotes the susceptance matrix whose elements are $B_{k l}=-Y_{k l}$ for the offdiagonal terms and

$$
B_{k k}=-\sum_{l \neq k} B_{k l}
$$

(the column sum of off-diagonals) for diagonal elements. $Y_{k l}$ denotes the admittance of the line between nodes $k$ and $l . B^{+}$is the Moore-Penrose pseudoinverse of $B$, and $B^{D}$ is a diagonal matrix with $B_{k k}^{D}=Y_{i j}$.

For further information on DC load flow models, see Oren et al (1995) and Contreras (1997).

To point out some properties of DC load flow models let us consider the example with 3 nodes (2 generators, nodes 1 and 3 and 1 consumer, node 2) and 3 transmission lines. In Figure 1 we can how line admittances determine the flows of the network. In 1(a) the flow is not constrained by transmission capacities.

If we double the admittance and maximal transmission capacity between the nodes 2 and 3 (which can be regarded as the physical duplication of the power line, shown on Figure 1), the line 1-3 will be overloaded, since the increased overall admittance of line 1-3-2 "draws the flow" onto that branch from 1-2. Of course a suitable reduction of production and consumption levels leads to a feasible flow as shown on Figure 1(c), but the example illustrates that transmission networks may exhibit Braess's paradox-like phenomena, where adding local capacities may decrease overall performance. While Braess's paradox is due to the inefficiency of the Nash equilibrium of a selfish traffic routing problem, which can be solved by a central planner, here, the production/consumption levels, network topology and line admittances uniquely determine the flows and the sole remedy is the reduction of these levels. 


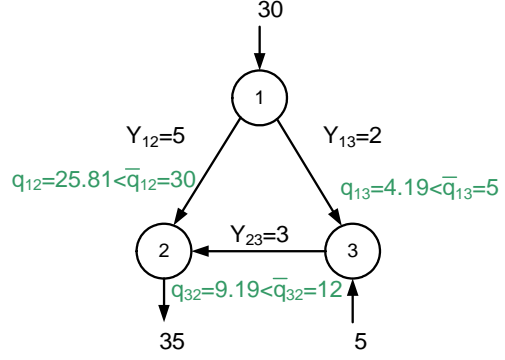

(a)

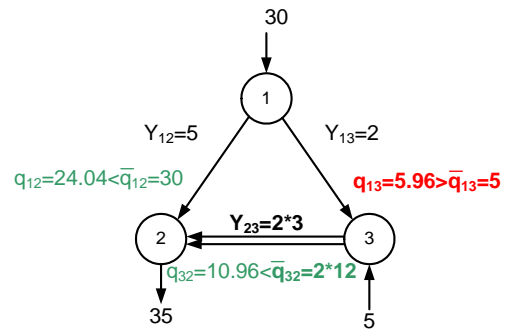

(b)

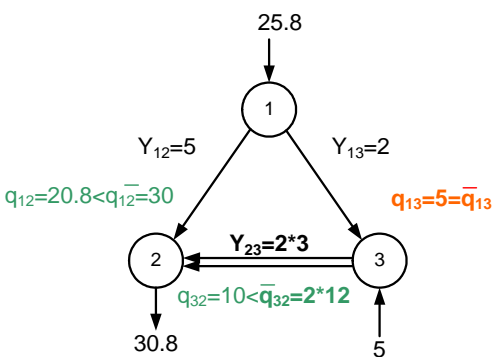

(c)

Fig. 1 Power flow on a simple 3-node network. If we double the connection between 2 and 3, line 1-3 gets overloaded. $Y$ denotes the line admittances. $q_{i j}$ denotes the actual power flow on edge $i, j$ while $\bar{q}_{i j}$ denotes the maximum transmission capacity.

Players of the game correspond to nodes (generators or consumers), while coalitions of the game correspond to balancing groups for which the total inlet and outlet power has to be equal. A central independent network regulator may limit the power in and outlet of the coalitions (in other words reschedule the generators and curtail the loads) in order to maximize the total power transmitted by the network and secure the safe operation of the system and avoid line overloads. As described in (Csercsik and Kóczy, 2011), maximization of the total transmitted power can be achieved by the solution of a linear programming problem, in which the objective function is the total power injected in the network, while inequality type constraints correspond to line loads and the maximal values of generation and consumption rates. Further equality constraints correspond to the balance of the coalitions (balancing groups) and the total balance of the network.

The value of a certain coalition in this setup is determined as twice the total transmission within the coalition.

In the current paper, based on the defined model, the safety-critical rescheduling of generators and load curtailment in the case of an instantaneous failure of a single transmission line is analyzed.

Electrical power transmission networks are safety critical systems, which have to be operated in a fault-tolerant manner. Assuming a basically safe 
operation, in the case of sudden line or generator failure, the flows in the network change instantaneously, potentially enhancing the vulnerability of the system. In such cases the rescheduling of the system is needed to prevent further, possibly more dramatic failures (Thanikachalam and Tudor, 1971; Kaltenbach and Hajdu, 1971). The problem of network uncertainties is relevant in the case of network problems of other nature as well (Li et al, 2009; Chen et al, 2013).

As foreshadowed, in this work we will consider only line failures. Regarding our DC load flow model defined in (Csercsik and Kóczy, 2011) this means, that in the case of basic operation, the power in and outlets of the nodes have to be determined in a way, that if any line instantaneously fails, none of the remaining lines may be overloaded. We will call this a safe state of the system. This network stability requirement may be included in the LP problem described in eq. 13 of (Csercsik and Kóczy, 2011), by adding further inequality type constraints, which describe transfer limitations under line failures.

Basically, in the case of a line failure, the system may loose this stability property (the failure of an additional more line may lead to network flows, which exceed the maximum capacity of a certain line, or lines). In this case the generators of the system need to be rescheduled and the loads are curtailed to bring the system in a safe state again. This must be done in a way which implies the minimal total change in generation and consumption values (Kaltenbach and Hajdu, 1971). In the following we will demonstrate the importance of the failure induced safety critical rescheduling on a simple 4-node network.

Example

Let us consider the network depicted in Figure 2.

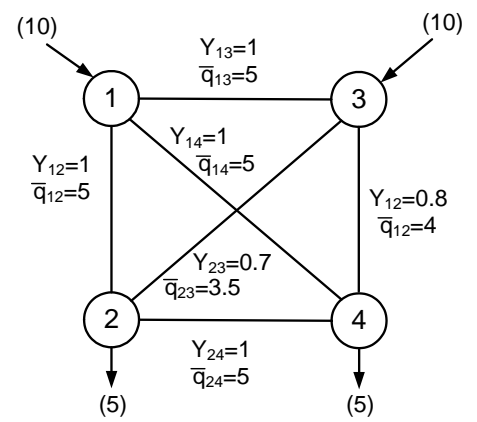

Fig. 2 The basic structure and parameters of the 4-node network. $Y_{i j}$ corresponds to the admittance value of the line between $i$ and $j$, while $\bar{q}_{i j}$ denotes the value of the maximal possible energy transfer on the corresponding line (power transmission capacity). The numbers in parentheses at the nodes correspond to maximal generating capacity and desired consumption.

If we assume the grand coalition to form, which implies only one equality type constraint corresponding to the balance of total inlet and outlet power, 
the a safe network configuration corresponding to maximal total transmission (which in this case satisfies all consumers) is depicted in Figure 3a.

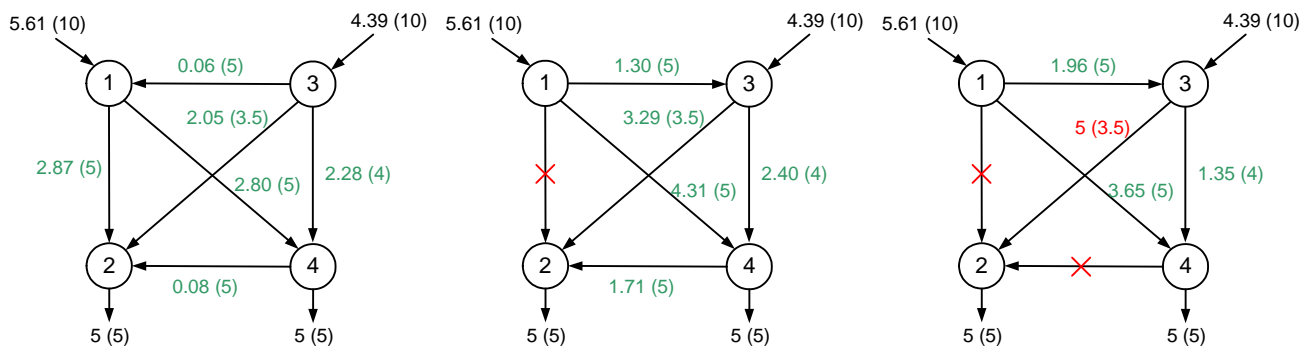

Fig. 3 Basic (safe) operation of the 4 node network without failures, flows without rescheduling in the case of the failure of line 1-2, and in the case of the failure of line 1-2 and 2-4: The first failure does not lead to line overload, but without rescheduling the second failure causes the overload of line $2-3$.

Since we assume a stable nominal operation, the line capacities are not exceeded in the case of the first failure (as depicted in Figure $3 \mathrm{~b}$ ), but without generator rescheduling and load curtailment after the second failure of line 2-4, line 2-3 is overloaded (which would lead to further failures). Via overheating of these lines, this may lead to further avalanche of failures and emergency shutdowns in critical case, which may lead to costs of billions of $\$ / E U R$ in most serious cases (Fairley, 2004). We assume that all generators can be rescheduled, and all loads can be curtailed - of course this is not the case in a realistic scenario, where some consumers are treated with priority in general. If we reschedule the generators and apply load curtailment after the first failure of line 1-2 in order to bring the network in safe state again (we do this under the constraint of implying minimal total change in generation values and loads), the second failure of the line 2-4 will not cause overload of any other lines as depicted in Figure 4.
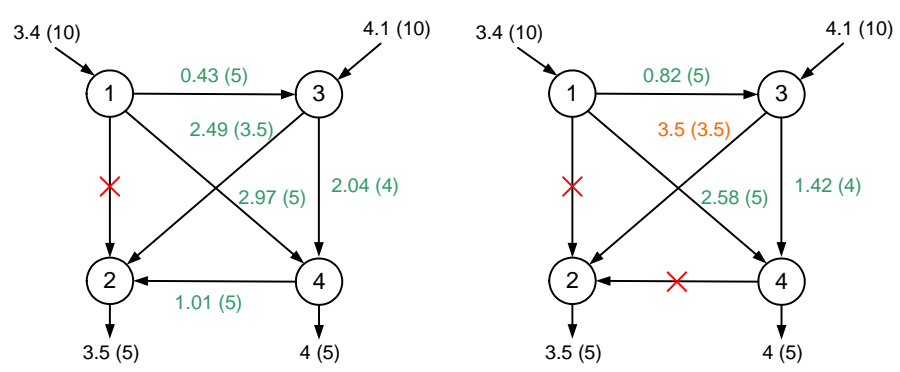

Fig. 4 Rescheduling of the network after the failure of line 1-2: The second failure of line 2-4 does not overload the remaining lines. Line 2-3 operating on the edge of his capacity shows, that the rescheduling of generators and the curtailment of loads was justified. 


\subsection{The Power Transmission Game with Safety Critical Rescheduling}

To define a cooperative game taking into account the uncertainty corresponding to the possible failure of lines, and the implied rescheduling which is causing changes in the transfer of certain coalitions, we use the formalism defined in Section 2.

In the case of $m$ lines, each of which may fail, we have $S=m+1$ number of states of nature in period $t=1$. The first state, $s=1$, corresponds to the normal (error-free) operation of the network, and the remaining $m$ states describe the possible failures of the corresponding $m$ lines. This implies that we will have $m+1$ state-dependent partition function form games.

We will demonstrate the calculation of the Sustainable Core in the case of the network depicted in Figure 2. The state-dependent minimal claim function corresponding to the normal operation and to the failures of certain lines, and the implied safety critical rescheduling are listed in Table 1. $s=1$ corresponds to the error-free operation of the network, while $s=2$ corresponds to the error of the line $1-2, s=3$ to the error of the line $1-3, s=4$ to the error of the line $1-4, s=5$ to the error of the line $2-3, s=6$ to the error of the line $2-4$ and $s=7$ to the error of the line 3-4.

Table 1 Minimal claim function; $v_{s}^{m c}$

\begin{tabular}{|c|c|c|c|c|c|c|c|}
\hline coalition & $s=1$ & $s=2$ & $s=3$ & $s=4$ & $s=5$ & $s=6$ & $s=7$ \\
\hline$\emptyset$ & 0 & 0 & 0 & 0 & 0 & 0 & 0 \\
$\{1\}$ & 0 & 0 & 0 & 0 & 0 & 0 & 0 \\
$\{2\}$ & 0 & 0 & 0 & 0 & 0 & 0 & 0 \\
$\{3\}$ & 0 & 0 & 0 & 0 & 0 & 0 & 0 \\
$\{4\}$ & 0 & 0 & 0 & 0 & 0 & 0 & 0 \\
$\{1,2\}$ & 10 & 7 & 10 & 7 & 10 & 7 & 9.58 \\
$\{1,3\}$ & 0 & 0 & 0 & 0 & 0 & 0 & 0 \\
$\{1,4\}$ & 10 & 8 & 10 & 8 & 9.97 & 8 & 10 \\
$\{2,3\}$ & 10 & 7 & 7 & 7 & 7.02 & 7 & 7 \\
$\{2,4\}$ & 0 & 0 & 0 & 0 & 0 & 0 & 0 \\
$\{3,4\}$ & 10 & 8 & 7 & 8 & 8 & 8 & 5.92 \\
$\{1,2,3\}$ & 10 & 7 & 10 & 10 & 10 & 7 & 10 \\
$\{1,2,4\}$ & 17.08 & 10 & 10 & 10 & 14.44 & 15 & 14.12 \\
$\{1,3,4\}$ & 10 & 10 & 10 & 8 & 10 & 8 & 10 \\
$\{2,3,4\}$ & 14.72 & 13 & 7 & 12 & 8 & 14.12 & 7 \\
$\{1,2,3,4\}$ & 20 & 15 & 17 & 15 & 18 & 15 & 17 \\
\hline
\end{tabular}

To evaluate the players' payoffs, we assume that all four players in our Example have the following utility function;

$$
u^{i}\left(x^{i}\right)=0.88\left(1-e^{-0.001 x_{1}^{i}}\right)+\sum_{s=2}^{s=7} 0.02\left(1-e^{-0.001 x_{s}^{i}}\right) \text { for all } i \in N
$$

One can interpret this expression as a von Neumann-Morgenstern utility function, where the probability of error-free operation is $88 \%$, and the probability of any line failing is $2 \%$. 
Now consider the following allocation:

$$
x=\left(\begin{array}{cccc}
2.91226 & 7.08774 & 2.91226 & 7.08774 \\
2 & 5 & 2 & 6 \\
5.7565 & 4.2435 & 2.7565 & 4.2435 \\
3 & 4 & 3 & 5 \\
5.51228 & 4.48772 & 3.21021 & 4.78979 \\
0 & 7 & 0 & 8 \\
5.53279 & 4.36166 & 2.63834 & 4.46721
\end{array}\right) \in \mathbb{R}^{S \times N}
$$

It is easy to show that this allocation belongs to the Sustainable Core of the above defined game. First, one can check that $x_{s} \in \mathrm{RC}\left(\Gamma_{s}\right)$ holds for all $s \in S$, thus there is no blocking possibility in time period $t=1$. Now we only need to see if Part $(b)$ of Definition 6 holds. We show next that $x$ maximizes the sum of the players' utilities over allocations $x^{C}$ with $x_{s}^{C} \in \operatorname{RC}\left(\Gamma_{s}^{C}\right)$ for all $s \in S$.

Consider the following constrained maximization problem,

$$
\begin{aligned}
\max _{x^{C}} & \sum_{i \in C} u^{i}\left(x^{i}\right) \\
\text { s.t. } & \sum_{i \in C} x_{s}^{i}=v_{s}^{m c}(C), \quad s \in S, \\
& \sum_{i \in D} x_{s}^{i} \geq v_{s}^{m c}(D), \quad s \in S, \emptyset \neq D \subsetneq C,
\end{aligned}
$$

where condition (2) is required for ex post efficiency and inequality (3) is a no-blocking condition. A solution to the maximization problem maximizes the sum of the players' utilities among those allocations that belong to $\mathrm{RC}\left(\Gamma_{s}^{C}\right)$ for all $s \in S$. Since the given allocation $x$ is a solution to this problem, it follows that there is no blocking possible in period $t=0$ either.

Hence, $x \in \mathrm{SC}(\Gamma)$.

It is worthwhile to note that this outcome is not really sensitive to the exact choice of the utility functions; e.g. the given $x$ would also be a solution if the utility functions were

$$
u^{i}\left(x^{i}\right)=0.4\left(1-e^{-0.01 x_{1}^{i}}\right)+\sum_{s=2}^{s=7} 0.1\left(1-e^{-0.01 x_{s}^{i}}\right) \text { for all } i \in N .
$$

This example demonstrates that using a cooperative game and the concept of the Sustainable Core may help us to find such stable solutions to such difficult problems as the safety-critical operation of the power transmission networks that take into account possible line failures as well. This solution may be used in reality to facilitate immediate reaction to possible failures, which might lead to disastrous outcomes if reaching an agreement after the failure would take any time. 


\section{Conclusion}

While in numerous real-life situations agreements have to be achieved in an environment with externalities and uncertainty about the future, the cooperative game theory literature has not considered this scenario yet. In this paper we introduced a new class of games, the partition function form games with uncertainty, to provide a framework to analyze these problems. We also proposed the Sustainable Core to solve the game, which is an extension of the Recursive Core and the Weak Sequential Core to this more complex setup.

As a possible application we analyzed the game of consumers and generators on a lossless DC load flow model of the electrical energy transmission network. While the described safety critical rescheduling of generators and curtailment of loads in the case of an arising line failure is a pure technological approach to avoid further overloads and shutdowns, dealing with its possibility in energy transmission contracts raises economical questions, for which the concept of the introduced sustainable core may serve as a possible solution concept. In this concept we assumed that the probabilities of line failures are known to each player of the game. A straightforward possible future scenario could be to analyze how asymmetric information affects the results. In most of the practical cases, regional generators have detailed information only about the network parameters in their area. Players who can obtain information about other areas may possibly effectively increase their bargaining potential and expected payoff compared to others.

The proposed framework may serve as a solution for many problems from environmental agreements to the formation of trading blocks or common pool resource allocation issues.

\section{References}

Abrell J, Weigt H (2012) Combining energy networks. Networks and Spatial Economics 12(3):377-401, DOI 10.1007/s11067-011-9160-0, URL http://dx.doi.org/10.1007/s11067-011-9160-0

Aumann RJ, Peleg B (1960) Von Neumann-Morgenstern solutions to cooperative games without side payments. Bulletin of the American Mathematical Society 66:173-179

Beccuti A, Demiray T, Andersson G, Morari M (2010) A lagrangian decomposition algorithm for optimal emergency voltage control. Power Systems, IEEE Transactions on 25(4):1769 -1779, DOI 10.1109/TPWRS.2010.2043749

Chander P, Tulkens H (1997) The core of and economy with multilateral environmental externalities. International Journal of Game Theory 26(3):379401

Chen B, Lam W, Sumalee A, Li Q, Shao H, Fang Z (2013) Finding reliable shortest paths in road networks under uncertainty. Networks and 
Spatial Economics 13(2):123-148, DOI 10.1007/s11067-012-9175-1, URL http://dx.doi.org/10.1007/s11067-012-9175-1

Contreras J (1997) A cooperative game theory approach to transmission planning in power systems. PhD thesis, University of California, Berkeley

Csercsik D, Kóczy LÁ (2011) Externalities in the games over electrical power transmission networks. IEHAS Discussion Papers 1125, Institute of Economics, Hungarian Academy of Sciences, Budapest, URL http://econ.core.hu/file/download/mtdp/MTDP1125.pdf

Ehrenmann A (2004) Equilibrium problems with equilibrium constraints and their application to electricity markets. Fitzwilliam College, Fitzwilliam 154

Fairley P (2004) The unruly power grid. IEEE Spectrum 41:22-27

Gabriel S, Kiet S, Balakrishnan S (2004) A mixed integer stochastic optimization model for settlement risk in retail electric power markets. Networks and Spatial Economics 4(4):323-345, DOI 10.1023/B:NETS.0000047111.94897.d4

Gillies DB (1959) Solutions to general non-zero-sum games. In: Tucker AW, Luce RD (eds) Contributions to the Theory of Games IV, no. 40 in Annals of Mathematics Studies, Princeton University Press, Princeton, pp 47-85

Habis H, Herings PJJ (2011a) Core concepts for incomplete market economies. Journal of Mathematical Economics 47(4-5):595-609

Habis H, Herings PJJ (2011b) Transferable utility games with uncertainty. Journal of Economic Theory 146(5):2126-2139, URL http://ideas.repec.org/a/eee/jetheo/v146y2011i5p2126-2139.html

Hines P, Apt J, Talukdar S (2009) Large blackouts in North America: Historical trends and policy implications. Energy Policy $37(12): 5249 \quad-5259, \quad$ DOI $10.1016 / \mathrm{j}$.enpol.2009.07.049, URL http://www.sciencedirect.com/science/article/pii/S0301421509005667

Kaltenbach J, Hajdu L (1971) Optimal corrective rescheduling for power system security. IEEE Transactions on power Apparatus and Systems 90:843851

Kleindorfer P, Wu DJ, Fernando C (2001) Strategic gaming in electric power markets. European Journal of Operational Research 130:156-168

Kóczy LÁ (2006) A recursive core for partition function form games. Research Memorandum RM/06/031, METEOR, Maastricht University, Maastricht

Kóczy LÁ (2007) A recursive core for partition function form games. Theory and Decision 63(1):41-51, DOI 10.1007/s11238-007-9030-x

Leuthold F, Weigt H, von Hirschhausen C (2012) A large-scale spatial optimization model of the european electricity market. Networks and Spatial Economics 12(1):75-107, DOI 10.1007/s11067-010-9148-1, URL http://dx.doi.org/10.1007/s11067-010-9148-1

Li ZC, Lam W, Wong S (2009) The optimal transit fare structure under different market regimes with uncertainty in the network. Networks and Spatial Economics 9(2):191-216, DOI 10.1007/s11067-007-9058-z, URL http://dx.doi.org/10.1007/s11067-007-9058-z

Metzler C, Hobbs B, Pang JS (2003) Nash-cournot equilibria in power markets on a linearized dc network with arbitrage: Formulations and properties. Net- 
works and Spatial Economics 3(2):123-150, DOI 10.1023/A:1023907818360

Moulin H (1985) The separability axiom and equal sharing methods. Journal of Economic Theory 36(1):120-148

Oggioni G, Smeers Y, Allevi E, Schaible S (2012) A generalized nash equilibrium model of market coupling in the european power system. Networks and Spatial Economics 12(4):503-560, DOI 10.1007/s11067-011-91667, URL http://dx.doi.org/10.1007/s11067-011-9166-7

Oren S, Spiller P, Varaiya P, Wu F (1995) Folk theorems on transmission access: Proofs and counter examples. Working papers series of the Program on Workable Energy Regulation (POWER) PWP-023, University of California Energy Institute 2539 Channing Way Berkeley, California 94720-5180, www.ucei.berkeley.edu/ucei

Predtetchinski A, Herings PJJ, Peters H (2002) The strong sequential core for two-period economies. Journal of Mathematical Economics 38:465-482

Ray D (1989) Credible coalitions and the core. International Journal of Game Theory 18:185-187

Ray D, Vohra R (1997) Equilibrium binding agreements. Journal of Economic Theory 73(1):30-78

Shenoy PP (1980) A dynamic solution concept for abstract games. Journal of Optimization Theory and Applications 32(2):151-169

Smeers Y (2003) Market incompleteness in regional electricity transmission. part ii: The forward and real time markets. Networks and Spatial Economics 3(2):175-196, DOI 10.1023/A:1023916120177

Thanikachalam A, Tudor J (1971) Optimal rescheduling of power for system reliability. Power Apparatus and Systems, IEEE Transactions on PAS90(5):2186 -2192, DOI 10.1109/TPAS.1971.293039

Thrall RM, Lucas WF (1963) $n$-person games in partition function form. Naval Research Logistics Quarterly 10(4):281-298

De la Torre S, Conejo A, Contreras J (2003) Simulating oligopolistic poolbased electricity markets: A multiperiod approach. Power Systems, IEEE Transactions on 18(4):1547-1555 